\title{
Globular domain of adiponectin: promising target molecule for detection of atherosclerotic lesions
}

This article was published in the following Dove Press journal:

Biologics:Targets and Therapy

6 September 201 I

Number of times this article has been viewed

\author{
Gunter Almer' \\ Matthias Saba-Lepek ${ }^{2}$ \\ Samih Haj-Yahya' \\ Eva Rohde ${ }^{3}$ \\ Dirk Strunk ${ }^{4}$ \\ Eleonore Fröhlich ${ }^{5}$ \\ Ruth Prass ${ }^{2}$ \\ Harald Mangge' \\ 'Clinical Institute of Medical and \\ Chemical Laboratory Diagnostics, \\ Medical University of Graz, ${ }^{2}$ Institute \\ of Biophysics and Nanosystems \\ Research, Austrian Academy of \\ Science, ${ }^{3}$ University Clinic of Blood \\ Group Serology and Transfusion \\ Medicine, ${ }^{4}$ University Clinic of \\ Hematology, ${ }^{5}$ Center for Medical \\ Research, Medical University of \\ Graz, Graz, Austria
}

Background: Adiponectin, an adipocyte-specific plasma protein, has been shown to accumulate in injured endothelial cells during development of atherosclerotic lesions. In this study, we investigated the potential of different adiponectin subfractions with special emphasis on globular adiponectin ( $\mathrm{gAd}$ ) to recognize and visualize atherosclerotic lesions.

Methods: Recombinant mouse gAd and subfractions of full-length adiponectin (ie, trimeric, hexameric, and oligomeric forms) were fluorescence-labeled. Aortas of wild-type and apoprotein E-deficient mice fed a high cholesterol diet were dissected and incubated with the labeled biomarkers. Imaging was performed using confocal laser scanning microscopy.

Results: Confocal laser scanning microscopic images showed that gAd binds more strongly to atherosclerotic plaques than full-length adiponectin subfractions. Further, we showed that gAd accumulates preferentially in endothelial cells and the fibrous cap area of plaques. Here we demonstrate for the first time that gAd recognizes atherosclerotic plaques on aortic sections of apoprotein E-deficient mice.

Conclusion: These results suggest that gAd, in addition to its physiological properties, is also suitable as a target molecule for prospective diagnostic strategies in imaging atherosclerotic lesions.

Keywords: adiponectin subfractions, atherosclerosis, fibrous cap, globular adiponectin, vascular imaging

\section{Introduction}

Accumulating evidence suggests that adipose tissue is a highly active endocrine organ ${ }^{1}$ producing potent anti-inflammatory proteins in its normal state. ${ }^{2}$ Probably the most important is adiponectin, ${ }^{3}$ which is predominantly synthesized by adipocytes. ${ }^{4}$ The basic structure of the full-length form of adiponectin (fAd) is a 247 amino acid protein with four domains, ie, an amino terminal signal sequence, a nonconserved variable region, a collagenous domain, and a carboxyterminal globular domain. ${ }^{5}$ In the bloodstream, adiponectin circulates at concentrations of $2-30 \mu \mathrm{g} / \mathrm{mL}$ of blood ${ }^{6}$ and in different molecular weight forms, named high molecular weight (oligomer), medium molecular weight (hexamer), and low molecular weight (trimer) adiponectin. ${ }^{7,8}$ Interesting to note is that these subfractions exert markedly different biological functions. ${ }^{9}$

Adiponectin is thought to protect against diabetes and atherosclerosis. ${ }^{10} \mathrm{Li}$ et al showed that local treatment with adiponectin reduces atherosclerotic plaque size in rabbits by decreasing the expression of vascular cell adhesion molecule-1 (VCAM-1) and intercellular adhesion molecule-1 (ICAM-1) in the vascular walls. ${ }^{11}$ Furthermore, hypoadiponectinemia was found to be a significant predictor of endothelial dysfunction
Correspondence: Harald Mangse Clinical Institute of Medical and Chemical Laboratory Diagnostics, Auenbruggerplatz 4, $8036 \mathrm{Graz}$, Austria Tel +4331638583340

Fax +433163854024

Email harald.mangge@klinikum-graz.at
(C) 20I I Almer et al, publisher and licensee Dove Medical Press Ltd. This is an Open Access article which permits unrestricted noncommercial use, provided the original work is properly cited. 
in both peripheral and coronary arteries. ${ }^{12,13}$ In subjects with metabolic syndrome, a significant negative correlation was observed between serum adiponectin concentrations, plaque volume, and highly sensitive C-reactive protein levels. ${ }^{14}$ Moreover, Waki et al showed that leukocyte elastase secreted from activated monocytes and/or neutrophils can cleave fAd. ${ }^{15}$ This cleavage might be the reason for the generation of the $17 \mathrm{kDa}$ globular fragment of adiponectin (gAd), which is found at lower levels (about 1\% of total adiponectin) in the circulation. ${ }^{16}$ gAd was shown to increase insulin-stimulated glucose uptake and to boost $\beta$-oxidation of fatty acids, ${ }^{17,18}$ while other functions remain controversial. ${ }^{19,20}$

Two main receptors are known to bind adiponectin, namely adipoR1 and adipoR2. ${ }^{21}$ AdipoR1 is expressed mainly on vascular smooth muscle cells and endothelial cells, predominantly binding gAd. AdipoR2 binds both gAd and fAd and is highly expressed on hepatocytes, and in smaller amounts in the hypothalamus and on brain endothelial cells. ${ }^{22}$ Furthermore, fAd was shown to bind to T-cadherin ${ }^{23}$ and to the multifunctional protein, calreticulin. ${ }^{24}$

With respect to the atherosclerotic scenario, adiponectin was found to accumulate in the subendothelial space of vascular walls when the endothelial barrier is injured. There it binds to different types of collagen present in the vascular intima. ${ }^{25}$ In contrast, adiponectin was not detected in the subendothelial space of human atherosclerotic lesions with an intact endothelium. ${ }^{26}$ Based on these observations, we hypothesized that distinct isoforms of adiponectin might show different binding affinities to atherosclerotic lesions in the vascular wall. We analyzed the potential of fluorescence-labeled gAd and fAd subfractions (fAd-Sfs) to bind to atherosclerotic lesions in apoprotein E-deficient mice. Consecutive cell culture studies were performed to identify target structures which might be responsible for specific bindings.

\section{Materials and methods \\ Reagents}

Recombinant mouse gAd raised in Escherichia coli and a monoclonal mouse antihuman adiponectin antibody (used as the primary antibody) were purchased from Atgen, Korea, and fAd-Sfs raised in human embryonic kidney cells were from Biovendor, Czech Republic. A rabbit antihuman collagen type I antibody (Rockland Immunochemicals, Philadelphia, PA) was used as a positive control, and nonspecific rat antihuman $\mathrm{IgG} 2 \mathrm{a}$ as a negative control, similar in molecular weight $(150 \mathrm{kDa})$ to the fAd-Sf hexamer (167 kDa). AlexaFluor488-prelabeled rat antimouse CD31 and rat antimouse CD68 antibodies were used as endothelial cell and macrophage markers, respectively (AbD Serotec, Dusseldorf, Germany). For further comparative staining experiments, mouse interleukin 10, raised in $E$. coli, from Genescript (Piscataway, NJ) was used because it has a similar molecular weight (19 kDa) to gAd (17 kDa). In each case, two anti-AdipoR1 and anti-AdipoR2 antibodies were purchased (rabbit antimouse antibodies, LifeSpan Biosciences, Seattle, WA; goat antimouse antibodies, Santa Cruz, CA), as well as a sheep antimouse calreticulin antibody (Abcam, Cambridge, UK). As a long wavelength emitting dye, Atto655 (ATTO-TEC GmbH, Siegen, Germany), was used either as a maleimide-functionalized label (Atto655Mal) or as an amine reactive carboxylic acid succinimidyl ester (Atto655-NHS). As a second dye, Alexa Fluor ${ }^{\circledR}$ 488 carboxylic acid succinimidyl ester (Alexa488-NHS, Invitrogen, Carlsbad, CA), was chosen to gain a reproducible differentiation in double-labeling experiments after incubation of the tissue with Atto655.

\section{Fluorescence labeling}

Labeling procedures followed the standard protocols recommended by the supplier of reactive fluorophores (http:// www.atto-tec.com). Slight alterations in molar ratios of dye and protein/antibody were performed to preserve the maximum binding efficiency to the target protein. In the case of gAd, labeling was performed with Atto655-Mal coupling to the single cysteine residue within the protein using a 20-fold molar excess of the dye under basic conditions in phosphate-buffered saline (10 mM, $150 \mathrm{mM} \mathrm{NaCl}, \mathrm{pH} 8.0$ ). Full-length subfractions of adiponectin, rat antihuman IgG2a, and rabbit and goat antimouse AdipoR2 antibodies were labeled with amine-reactive Atto655-NHS, labeling the side chains of lysine residues. For the labeling of the fAd-Sfs and interleukin 10, a 2-molar excess of Atto655-NHS referred to the hexamer (fAd-Sfs) and monomer (interleukin 10) content was used. Rat antihuman IgG2a and antimouse AdipoR2 antibodies were labeled under equimolar conditions with Atto655-NHS. Rabbit antihuman collagen type I, rabbit and goat antimouse AdipoR1, and sheep anticalreticulin antibodies were labeled with Alexa488-NHS also using equimolar dye/protein ratios. All NHS-based labeling procedures were performed in $150 \mathrm{mM}$ bicarbonate buffer containing $5 \mathrm{mM}$ ethylenediamine tetra-acetic acid at $\mathrm{pH}$ 8.3. Labeling was carried out at room temperature followed by extensive dialysis at $4^{\circ} \mathrm{C}$ to stop the reaction and remove nonbound dye. Dialysis was performed in microdialysis tubes with a molecular weight cutoff from 5-50 kDa depending on the molecular mass of the labeled protein. 


\section{SDS-PAGE and Western blot analysis}

Sodium dodecyl sulfate polyacrylamide gel electrophoresis (SDS-PAGE) techniques were used for the detection of all fluorescence-labeled adiponectin isoforms, antibodies, and unspecific IgG. All additional reagents were purchased from Bio-Rad Laboratories, München, Germany. Labeled and native proteins were diluted with double-distilled water and mixed in a ratio of 1:1 with Laemmli sample buffer. Neither heat denaturation nor $\beta$-mercaptoethanol was applied. gAd was then applied on an $18 \%$ gel. fAd-Sfs, unspecific IgG, and all antibodies were applied on $10 \%$ Tris- $\mathrm{HCl}$ precast gels. A broad range prestained SDS-PAGE standard was used for the size mapping of all protein fractions. After fluorescence imaging of the labeled proteins excited at $\lambda_{\text {flu }}=750 \mathrm{~nm}$ (Maestro In Vivo Imaging System ${ }^{\mathrm{TM}}, \mathrm{CRi}$, Woburn, MA), the gels were control stained with Bio-Safe ${ }^{\mathrm{TM}}$ Coomassie G250 stain.

Western blotting was used for examination of possible steric changes in gAd and fAd-Sfs due to the fluorescencelabeling process. Samples from unstained precast gels were blotted to polyvinylidene difluoride membranes. Immunoblots were incubated with the primary monoclonal mouse antihuman adiponectin antibody, detected using a horseradish peroxidase-conjugated goat antimouse secondary antibody, and visualized by the chemoluminescence reaction with the SuperSignal ${ }^{\circledR}$ West Femto maximum sensitivity substrate (all from Pierce Protein Research Products, Rockford, IL).

During the labeling reaction for gAd, small amounts of gAd dimers and trimers were formed which could be detected by Coomassie staining and Western blotting, respectively (Figure 1A). All fAd-Sfs were labeled quantitatively, whereby a shift from the dominant hexameric form in the unlabeled state to the monomeric, trimeric, and oligomeric fractions during the reaction was detected by Coomassie staining. Western blotting confirmed the detection of all fAd-Sfs and, most strongly, the hexameric and oligomeric forms (Figure 1B).

\section{Animal experiments}

All animal experiments were approved by the Ministry of Science and Research, Austria. Apoprotein E-deficient mice with a $\mathrm{C} 57 \mathrm{~B} 1 / 6 \mathrm{~J}$ genetic background (Charles River Laboratories, Brussels, Belgium) were fed starting at the age of 4 months with a western-type (21\% XL) experimental food (Ssniff Spezialdiaeten GmbH, Soest, Germany) for 2-3 months. Age-matched wild-type mice (Medical University of Vienna, Austria) were used as a control strain. Due to the fact that no gender-specific differences during the staining trials were observed, the experiments were

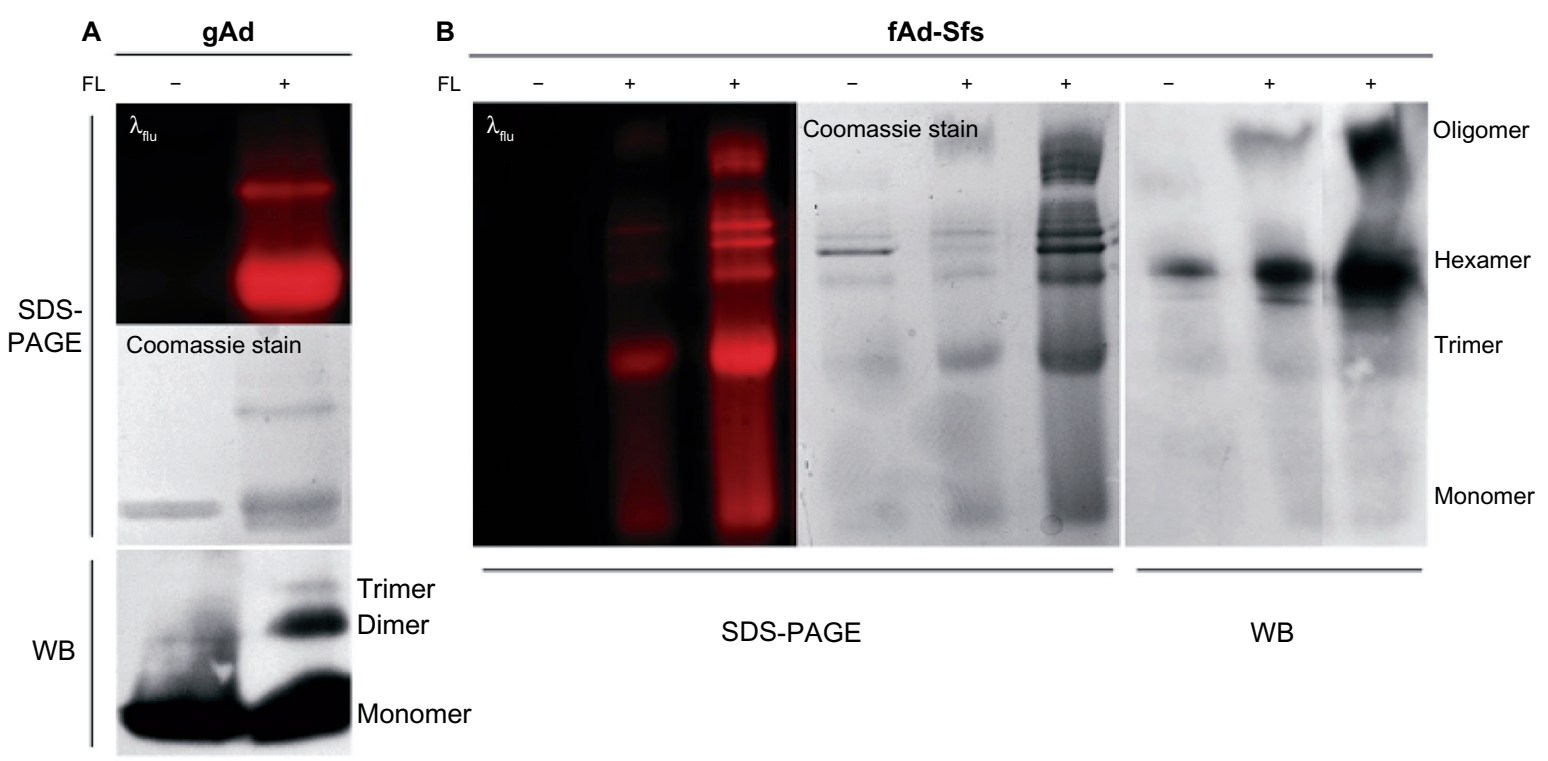

Figure I Atto655 labeling of adiponectin isoforms. Free dye was eliminated by dialysis. Sodium dodecyl sulfate polyacrylamide gel electrophoresis (SDS-PAGE) visualization was performed by fluorescence imaging and shows the signals of the fluorescence-labeled proteins. Protein size was determined by comparison with a broad range of prestained SDS-PAGE standards (Bio-Rad Laboratories, München, Germany). Identification of the proteins was verified using a monoclonal mouse antihuman adiponectin antibody, indicating that no steric changes occurred during the labeling reaction. (A) The sole cysteine residue of globular adiponectin was labeled with the dye via a maleimide group. $5 \mu \mathrm{g}$ of the unlabeled and $10 \mu \mathrm{g}$ of the labeled globular adiponectin was applied for polyacrylamide gel electrophoresis, half thereof for Western blotting analysis. (B) Lysines of the full-length adiponectin subfractions were labeled with the dye in a molar ratio of I:2 (corresponding to the full-length adiponectin subfraction hexameric form) via NHS ester bonds. $2 \mu \mathrm{g}$ of the unlabeled and $2 \mu \mathrm{g}$ and $10 \mu \mathrm{g}$ of the labeled full-length adiponectin subfractions were applied for polyacrylamide gel electrophoresis, a quarter thereof for Western blotting analysis.

Abbreviations: FL, fluorescence label; WB, Western blot. 
performed independent of gender. Injured endothelium was defined as the activated endothelial layer covering the atherosclerotic lesions of apoprotein E-deficient mice. For each ex vivo staining trial, three mice were randomly sacrificed with an overdose of isoflurane (Abbott Ges $\mathrm{mbH}$, Wien, Austria), the chest opened, and the circulation immediately rinsed with phosphate-buffered saline ( $\mathrm{pH} 7.4)$ for 15 minutes by perfusing the heart with an injection needle and transecting the vena cava. The aorta, including the aortic arch, were dissected, cut open, washed with phosphatebuffered saline ( $\mathrm{pH}$ 7.4) and transferred to Krebs-Henseleit solution (118 mM NaCl; $25 \mathrm{mM} \mathrm{NaHCO}_{3} ; 2.8 \mathrm{mM} \mathrm{CaCl}_{2} 2$ $\mathrm{H}_{2} \mathrm{O} ; 1,17 \mathrm{mM} \mathrm{MgSO}_{4} 7 \mathrm{H}_{2} \mathrm{O} ; 4,7 \mathrm{mM} \mathrm{KCl} ; 1.2 \mathrm{mM} \mathrm{KH}_{2} \mathrm{PO}_{4}$; $2 \mathrm{mg} / \mathrm{mL}$ glucose; $\mathrm{pH} 7.4$ ) to maintain physiological activity of the tissue, and coevally blocked with $1 \%$ bovine serum albumin (Sigma-Aldrich, St Louis, MO) for 1 hour at room temperature. For control experiments, aortic sections were preincubated to excess with native $\operatorname{gAd}(60 \mu \mathrm{g} / \mathrm{mL})$ for 1 hour at $4^{\circ} \mathrm{C}$ to saturate possible adiponectin-binding regions. The samples were then incubated with the fluorescence-labeled proteins or antibodies ( 3 or $20 \mu \mathrm{g} / \mathrm{mL}$ of fluorescence-labeled $\mathrm{gAd} ; 20 \mu \mathrm{g} / \mathrm{mL}$ of unspecific rat antihuman IgG2a, rabbit antihuman collagen type I, or sheep antimouse calreticulin antibody; $30 \mu \mathrm{g} / \mathrm{mL}$ of fAd-Sfs; $10 \mu \mathrm{g} / \mathrm{mL}$ of interleukin $10 ; 50 \mu \mathrm{g} / \mathrm{mL}$ of rabbit or goat antimouse adipoR1 or antiadipoR2 antibody) for $1-2$ hours at $37^{\circ} \mathrm{C}$, with shaking in the dark to avoid fluorochrome bleaching. Hoechst 33342 fluorescence dye ( $1 \mu \mathrm{g} / \mathrm{mL}$, Invitrogen) was added to stain the cell nuclei. The aortic sections were then washed 3-4 times in phosphate-buffered saline (pH 7.4), put on a glass slide, covered with polyvinyl alcohol mounting medium with 1,4diazabicyclo[2.2.2] octane (Sigma-Aldrich), adjusted under a stereomicroscope, and squeezed with a cover slip.

\section{In vitro studies}

Human endothelial colony-forming cells were cultured in endothelial growth medium (EGM-2, Lonza, Walkersville, MD) supplemented with $10 \%$ fetal bovine serum. ${ }^{27}$ Selected cultures were treated with $30 \mu \mathrm{g} / \mathrm{mL}$ acetylated low density lipoprotein to apply lipid stress by changing the medium once a day. Control cultures were treated in the same manner, but were not exposed to acetylated low density lipoprotein. On day 7 , the cells were stained with $10 \mu \mathrm{g} / \mathrm{mL}$ Atto655-labeled gAd for 1 hour at $37^{\circ} \mathrm{C}$ and washed with medium.

The endothelial colony-forming cells used comply with American Heart Association guideline requirements. The cells are defined by the cell surface markers CD31+/CD34+/ CD45-/CD115-, lack hematopoietic potential, contribute to de novo blood vessel formation in vivo, and demonstrate clonal proliferative potential. ${ }^{28}$ Differentiation into endothelial cells was demonstrated by upregulation of genes/proteins not previously expressed by the experimental cell population, but normally expressed by primary endothelial cells and not expressed by other cell lineages. ${ }^{29}$

\section{Fluorescence imaging}

All fluorescence and transmitted light images of the aortic samples (Z-Stack images) and cultured cells were acquired using the LSM 510 META Axiovert 200M Zeiss confocal system (Jena, Germany), operating with a $25 \mathrm{~mW}$ laser diode tuned to $405 \mathrm{~nm}$ for Hoechst staining of the cell nuclei, a $30 \mathrm{~mW}$ argon laser tuned to $488 \mathrm{~nm}$ for visualization of the AlexaFluor488-labeled antibodies, and a $5 \mathrm{~mW} \mathrm{HeNe}$ laser tuned to $633 \mathrm{~nm}$ for visualization of all Atto655 labeled proteins. Images were collected using a $40 \times$ Plan-Neofluar 1.3 DIC oil immersion objective and a multitrack configuration, whereby the Hoechst, AlexaFluor488, and Atto655 signals were sequentially collected with BP $420-480 \mathrm{~nm}$, BP 505-550 nm, and BP 679-743 nm filters after excitation with $405 \mathrm{~nm}, 488 \mathrm{~nm}$, and $633 \mathrm{~nm}$ laser lines, respectively. In the sequential acquisitions for all three channels, the Zeiss AIM software version 4.2 was used. All confocal images were acquired with a frame size of $1024 \times 1024$ pixels averaged three times.

\section{Results}

\section{Globular adiponectin accumulates selectively in the outer layers of atherosclerotic plaques}

The binding affinity of gAd was evaluated for imaging of atherosclerotic lesions. Preparations from the inner aortic surface of apoprotein E-deficient and C57B16/J wild-type mice were imaged by confocal laser scanning microscopy, either unstained or stained with $20 \mu \mathrm{g} / \mathrm{mL}$ Atto655 fluorescence-labeled recombinant mouse gAd (gAd-Atto655). Sections from apoprotein E-deficient mice showed abundant atherosclerotic plaques of different sizes (Figure 2A, left panel), while aortic sections from the control mice showed neither atherosclerotic lesions nor a fluorescence signal of gAd-Atto655 (insert, right panel in Figure 2A). In contrast, a strong signal was detected on all atherosclerotic plaques from apoprotein E-deficient mice (Figure 2A, middle panel), while the surrounding surface area showed no fluorescence signal (Figure 2A, right panel). Moving from the top of a plaque down to the surrounding 

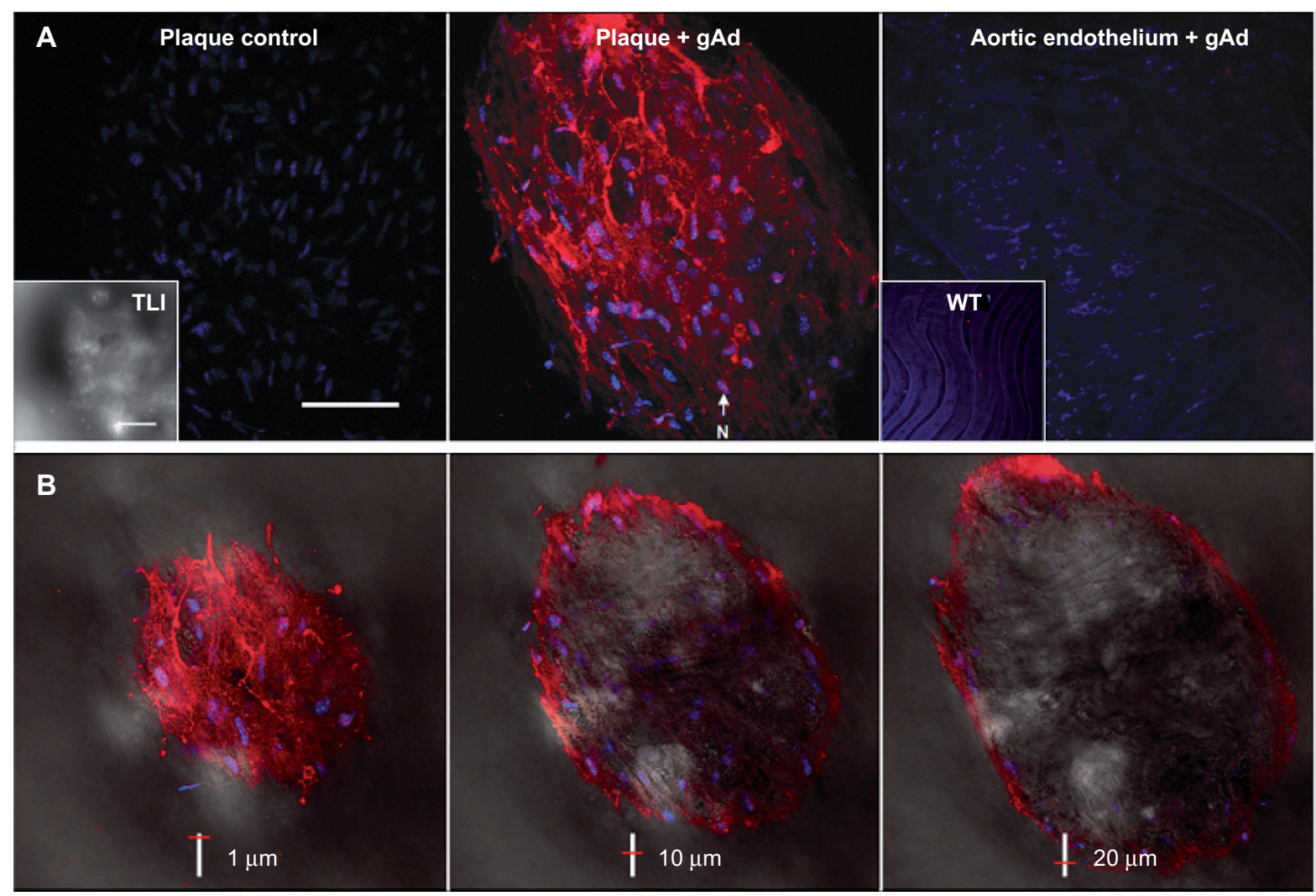

Figure 2 Globular adiponectin accumulates in the outer layer of atherosclerotic plaques. Aortic sections of apoprotein E-deficient and wild-type mice were incubated with globular adiponectin-Atto655 $20 \mu \mathrm{g} / \mathrm{mL}$, except for control sections. Sections were squeezed between a glass slide and a cover slip and visualized by confocal laser scanning microscopy (fluorescence and transmitted light). (A) Optical sections from aortic preparations projected into one plane. For each image, a series of 20-30 fluorescence images in Z (I-2 $\mu \mathrm{m}$ consecutive intervals) were projected in a single image. The nuclei (N) were stained with Hoechst (blue). Control plaque (left panel) and accumulation of globular adiponectin (red) on atherosclerotic plaques (middle panel) are shown; globular adiponectin does not bind to the intact aortic endothelium of apoprotein-E or wild-type mice (right panel). (B) Overlay of single Z-plane fluorescence and transmitted light images from a globular adiponectin-Atto655-stained plaque at different relative depth, as indicated on the left side bar in each panel are shown.

Note: Scale bars indicate $50 \mu \mathrm{m}$.

Abbreviations: WT, wild type; TLI, transmitted light image.

surface, the Z-Stack images revealed that gAd-Atto655, in contrast with the Hoechst nucleus dye, did not penetrate into the lipid core but stained the outer layer of the plaques (Figure 2B).

To support our results, costaining experiments were performed. Double-staining of the lesions confirmed that gAd is localized inside the endothelial cells of atherosclerotic plaques. Endothelial cells were identified using an AlexaFluor488-labeled antibody against the endothelial cell marker CD31 (Figure 3A). In another double-staining experiment using an AlexaFluor488-labeled antibody against collagen type I, colocalization of gAd with collagen type I was observed (Figure 3B).

To exclude nonspecific binding of the fluorescent dye to the plaque, some aortic sections were preincubated with a large amount of unlabeled $\mathrm{gAd}$. The sections were then stained with gAd-Atto655 as usual. On confocal laser scanning microscopy, we saw that the gAd-Atto655 signal from the atherosclerotic plaques of the presaturated aortic sections was significantly reduced compared with the nonsaturated sections (Figure 3C). We used Atto655-labeled mouse interleukin 10 for further comparative staining experiments because of its molecular weight being similar to gAd (gAd $17 \mathrm{kDa}$ versus interleukin $1019 \mathrm{kDa}$ ), and observed a different staining pattern with confocal laser scanning microscopy. As seen in Figure 3D, interleukin 10 penetrated the atherosclerotic plaques and did not preferentially accumulate in the outer layer, as did gAd.

\section{Binding efficiency of fAd-Sfs lower than of gAd for atherosclerotic plaques}

The atherosclerotic plaque binding efficiency of gAd was then compared with that of the fAd subfractions. Aortic sections from apoprotein E-deficient and wild-type mice were stained with either $30 \mu \mathrm{g} / \mathrm{mL}$ Atto655 fluorescence-labeled recombinant mouse fAd-Sfs (fAd-Sfs-Atto655) or $3 \mu \mathrm{g}$ 


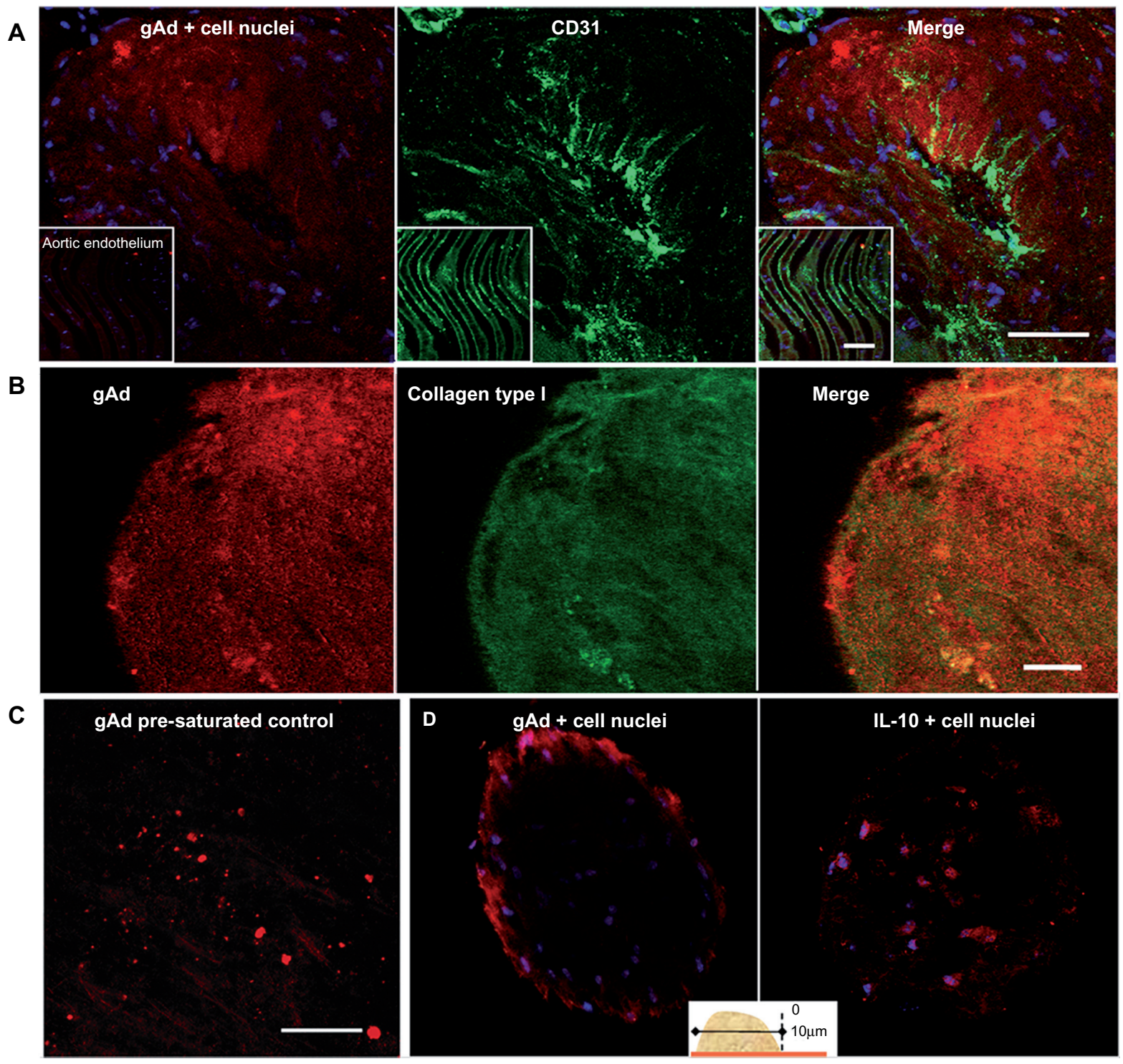

Figure 3 Globular adiponectin is localized in the fibrous cap of atherosclerotic plaques. (A) For double-staining experiments, aortic sections of apoprotein E-deficient mice were coincubated with globular adiponectin-Atto655 $20 \mu \mathrm{g} / \mathrm{mL}$ and AlexaFluor488-labeled rat antimouse CD3I antibody. The nuclei were stained with Hoechst (blue). Single Z-plane fluorescence images were taken at a depth of $4 \mu \mathrm{m}$ from the top of an atherosclerotic plaque showing the localization of globular adiponectin-Atto655 in the endothelial layer. The aortic surface is shown in the small inserts. (B) Aortic sections of apoprotein E-deficient mice were coincubated with globular adiponectin-Atto655 and AlexaFluor488-labeled rabbit antihuman collagen type I antibody. Single Z-plane fluorescence images were taken at a depth of $7 \mu \mathrm{m}$ from the top of an atherosclerotic plaque, showing colocalization of globular adiponectin and collagen type I. (C) For negative control experiments, aortic sections were preincubated with a high amount of native globular adiponectin. A stack of optical sections from an atherosclerotic plaque projected into one plane showed only a weak globular adiponectin-Atto655 signal, compared with the plaque image in the left panel in B. (D) Single Z-plane fluorescence light images from a globular adiponectin-Atto655 and an interleukin I0-Atto655-stained plaque at a depth of $10 \mu \mathrm{m}$ from top of the plaque. Images show the different staining patterns between globular adiponectin and interleukin 10 .

Notes: Scale bars: A and C, $50 \mu \mathrm{m}$; B, $20 \mu \mathrm{m}$.

gAd-Atto655. We found that the aortic surface surrounding the atherosclerotic plaques showed no signal after staining with fAd-Sfs-Atto655, while the plaques themselves showed a weak signal (Figure 4, middle panel). However, the staining signal was very faint compared with the signal obtained from gAd-Atto655. Even at equimolar concentrations, evaluated in relation to the $167 \mathrm{kDa}$ fAd hexamer, gAd showed the better staining signal (Figure 4, right panel). The rat IgG negative control showed no staining at all (Figure 4, left panels).
To identify the atherosclerotic plaque regions, the same aortic sections were costained with $5 \mu \mathrm{g} / \mathrm{mL}$ of a ready-labeled antibody against the macrophage marker CD68, shown in the other small inserts in Figure 4.

\section{High binding efficiency of gAd to atherosclerotic plaque surface}

The expression of known adiponectin receptors in the atherosclerotic plaque scenario was examined to look for specific 


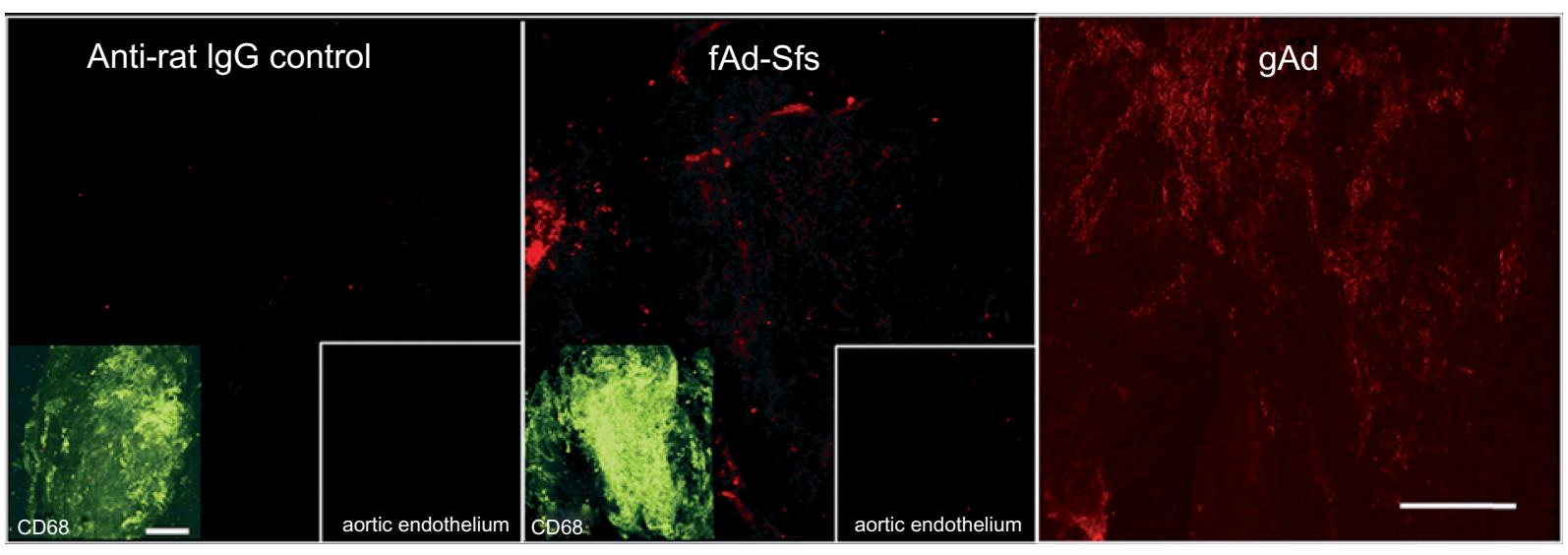

Figure 4 Binding efficiency of the full-length adiponectin subfractions to atherosclerotic plaques is much lower than that of globular adiponectin. Aortic sections of apoprotein E-deficient mice were incubated with unspecific rat antihuman lgG2a labeled with Atto655 as negative control (left) or full-length adiponectin subfraction-Atto655 $30 \mu \mathrm{g} / \mathrm{mL}$ (middle) or globular adiponectin-Atto655 $3 \mu \mathrm{g} / \mathrm{mL}$ (right). The inserts shown in the left and middle panels on the left side are positive controls, staining atherosclerotic plaques with an AlexaFluor488-prelabeled antibody against the macrophage marker CD68.

Note: Scale bars: $50 \mu \mathrm{m}$.

interactions of gAd with the atherosclerotic plaque surface. Aortic sections of apoprotein E-deficient mice were stained with fluorescence-labeled antibodies against AdipoR1 and AdipoR2, and against calreticulin.

While the gAd staining signal was detected at the surface of atherosclerotic plaques (Figure 5A and B, left panels), the antibodies against the gAd binding proteins, adipoR2 and calreticulin, showed staining signals from under the surface of the plaques (Figure 5A and B, middle and right panel). Thus, no colocalizations between gAd and these adiponectin-binding domains could be shown. Neither of the two antiadipoR1 antibodies generated a staining signal, either on the plaque surface or below (data not shown).

\section{Globular adiponectin accumulation in lipid-stressed endothelial colony-forming cells}

To understand the results from the staining of aortic mouse sections better, fluorescence-labeled gAd was evaluated in in vitro studies. Human endothelial colony-forming cells were cultured under proangiogenic and lipid stress conditions. The cells were incubated with $10 \mu \mathrm{g} / \mathrm{mL}$ of the same recombinant

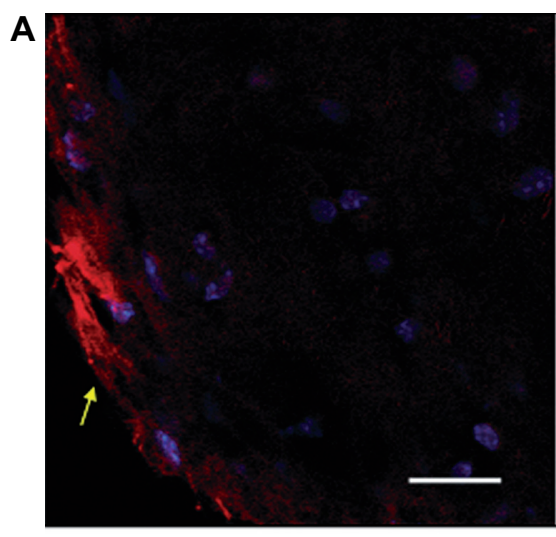

gAd

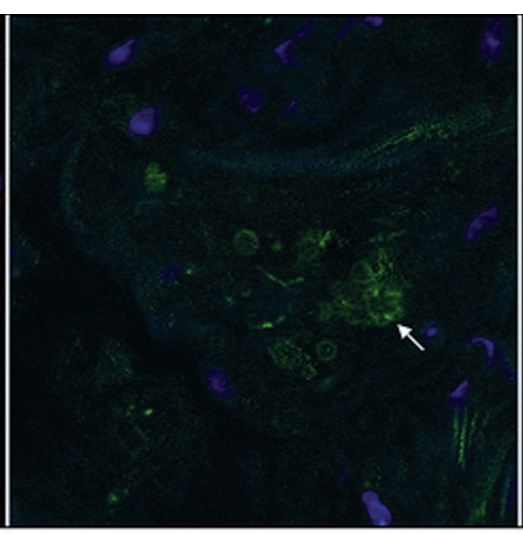

AdịpoR2

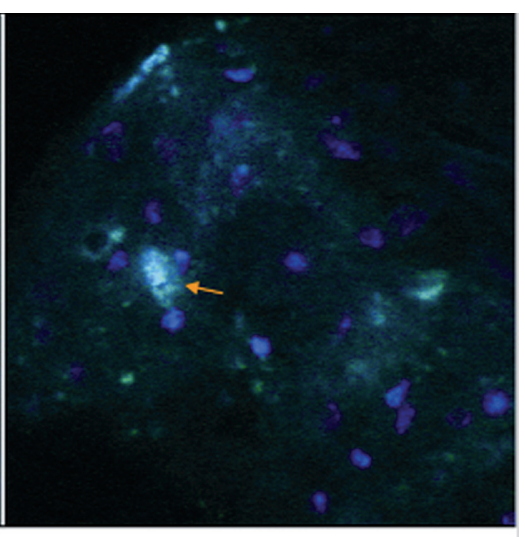

Calreticulin
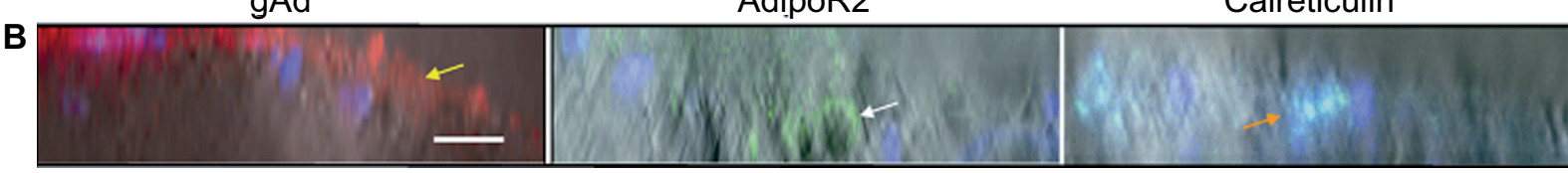

Figure 5 High binding efficiency of globular adiponectin to atherosclerotic plaques is independent of interactions with known adiponectin receptors. Aortic sections of apoprotein E-deficient mice were incubated with gAd-Atto655 $20 \mu \mathrm{g} / \mathrm{mL}$ (left panel) or fluorescence-labeled rabbit antimouse adipoRI and adipoR2 antibody (middle), or sheep antimouse calreticulin antibody (right). (A) Single horizontal Z-plane fluorescence images from atherosclerotic plaques. (B) Overlay of vertical fluorescence and transmitted light images. Yellow arrows indicate plaque surface globular adiponectin staining; white arrows indicate plaque interior adipoR2 staining; orange arrows indicate plaque interior calreticulin staining. Anti-adipoRI showed no staining signal (data not shown).

Note: Scale bars: A, $20 \mu \mathrm{m}$; B, $10 \mu \mathrm{m}$. 
mouse gAd-Atto655 as used for the aortic sections. An amino acid sequence alignment of mouse gAd with human gAd showed $91 \%$ identity and no gaps between amino acid sequences. Endothelial colony-forming cells, either cultured under endothelial cell culture conditions or treated with acetylated low density lipoprotein, did not bind gAd (Figure 6). Only a subset of acetylated low density lipoprotein-treated endothelial colony-forming cells showed an accumulation of gAd, which is marked by the yellow arrows in Figure 6. No signal was detected in cells treated with unspecific rat IgG (insert shown in Figure 6).

\section{Discussion}

Identification of atherosclerotic disease in its early stages and the need to follow the progression of atherosclerosis is still a challenge for medical imaging, which is limited not only by the properties of present imaging techniques, but also by the availability of specific biomarkers. ${ }^{30}$ In the present study, adiponectin was evaluated as a promising new target sequence. We showed for the first time that gAd, compared with fAd-Sfs, has higher binding efficiency for atherosclerotic plaques in apoprotein E-deficient mice, but not for the area surrounding the atherosclerotic plaques. Localization of gAd in endothelial cells and colocalization with collagen type I were observed, but no colocalizations of gAd with known adiponectin receptors or adiponectin binding proteins were found, pointing to a thus far unknown binding reaction.

Further aortic staining experiments showed that gAd generated staining patterns at atherosclerotic plaques markedly different from those of interleukin 10, although both gAd and interleukin 10 have approximately the same molecular weight and were labeled with the same fluorescence dye. Thus, the observed staining patterns at atherosclerotic plaque structures were generated by the proteins and not by an unspecific binding reaction of the fluorescence dye. Cell culture experiments, which should characterize its binding affinity in more detail, showed that gAd was internalized by endothelial colony-forming cells, but only the subset which was obviously injured by acetylated low density lipoprotein treatment. Based on our observations, compared with the
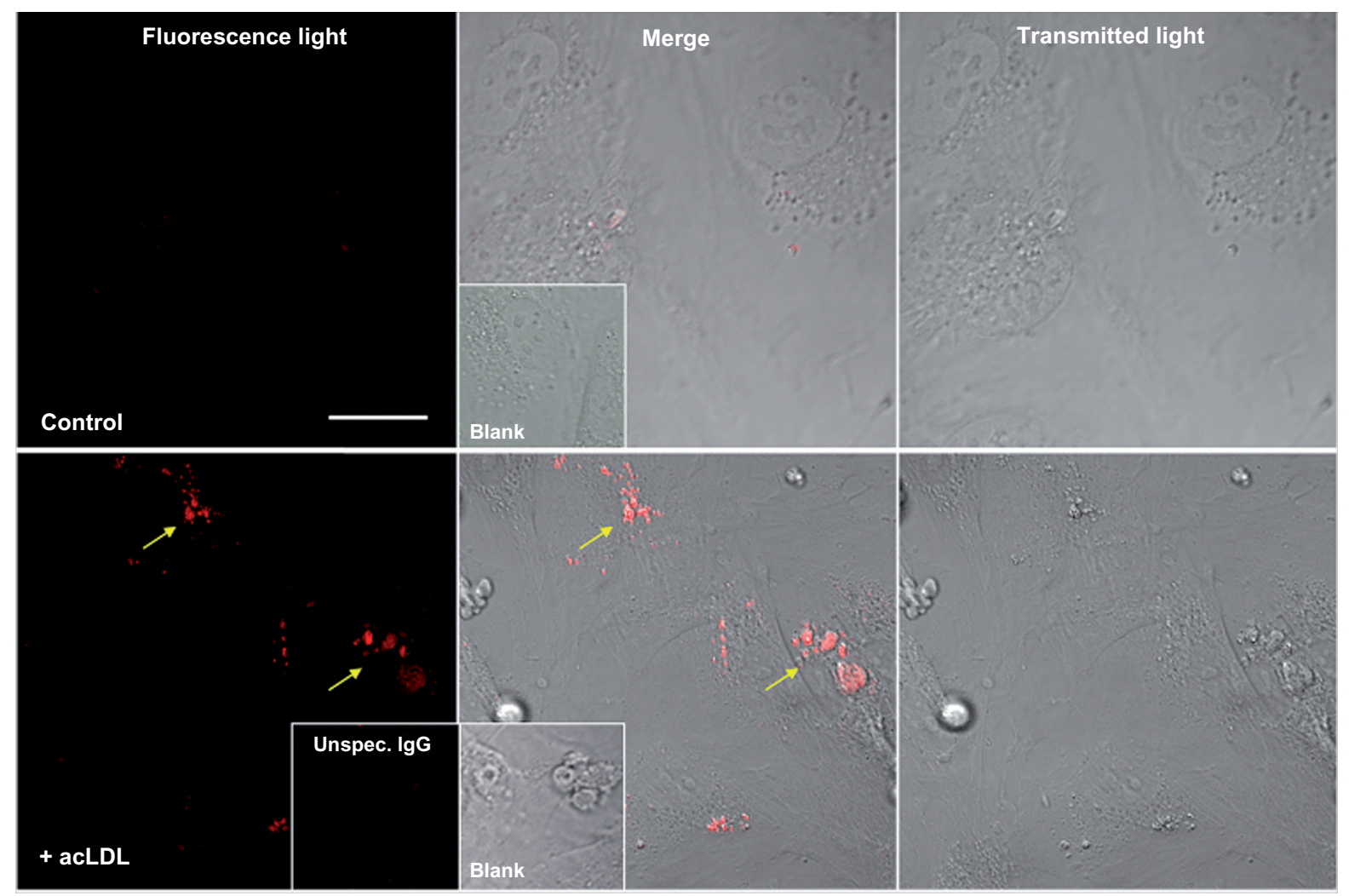

Figure 6 Globular adiponectin accumulates in lipid-stress injured human endothelial colony forming cells. Endothelial colony forming cells were cultured in the presence or absence (control) of acetylated low density lipoprotein and incubated with $10 \mu \mathrm{g} / \mathrm{mL}$ globular adiponectin-Atto655 or nonspecific IgG-Atto655 for I hour at $37^{\circ} \mathrm{C}$. Imaging was performed by confocal laser scanning microscopy. Unstained (blank) samples are shown as inserts in the middle panels. Yellow arrows indicate globular adiponectin accumulations. Note: Scale bar: $25 \mu \mathrm{m}$.

Abbreviations: acLDL, acetylated low-density lipoprotein; unspec., unspecific. 
data of Ouchi et $\mathrm{al}^{26}$ who showed that adiponectin was only detected at the injured atherosclerotic vascular wall, we hypothesize that the globular fragment of the total circulating amount of adiponectin can accumulate preferentially in atherosclerotic plaques with a thick fibrous cap.

Both anti-inflammatory actions and proinflammatory mechanisms have been described with reference to gAd. Recently, Kamio et al showed that gAd pretreatment of RAW264 cells significantly inhibited lipopolysaccharideinduced tumor necrosis factor alpha (TNF- $\alpha$ ) expression and enhanced the expression of interleukin $10 .{ }^{31}$ Whereas a study by Zhang et al showed that gAd suppresses TNF- $\alpha$ induced ICAM-1 expression in vascular endothelial cells at concentrations higher than $1 \mu \mathrm{g} / \mathrm{mL}$, the suppressive effect is lost at concentrations lower than $0.25 \mu \mathrm{g} / \mathrm{mL} .{ }^{32}$

In support of the proinflammatory actions of gAd, Hattori et al showed that $\mathrm{gAd}$ activates $\mathrm{NF}_{\mathrm{K}} \mathrm{B}$ in vascular endothelial cells, which induces the expression of proinflammatory and adhesion molecule genes. ${ }^{33}$ gAd increased the expression of 187 genes, including ICAM-1, VCAM-1, monocyte chemoattractant protein-1, and E-selectin, ${ }^{33}$ and also increased the expression of interleukin $6^{34}$ and granulocyte colony-stimulating factor.

Within this controversial scenario, we agree with the concept of Sun et $\mathrm{al}^{19}$ that the balance between proinflammatory and anti-inflammatory effects might depend on the concentration of gAd. While high concentrations of gAd may be proinflammatory, moderately high or low concentrations could act in an anti-inflammatory manner.

Shapiro and Scherer showed that the structure of gAd revealed an unexpected homology with the TNF family. Hence, we speculate that the homology between gAd and TNF- $\alpha$ may be a reason for the proinflammatory effects of abundant amounts of $\mathrm{gAd}$, because it is known that gAd, like TNF- $\alpha$, strongly activates $\mathrm{NF}_{\mathrm{K}} \mathrm{B} \cdot{ }^{36}$ Otherwise, gAd may reduce TNF- $\alpha$ expression ${ }^{37}$ due to a competitive reaction based on the similar structure of both proteins, a theory which should be elucidated in further experiments.

Little is known about the distribution of adiponectin receptors in endothelial and smooth muscle cells, especially in atherosclerotic regions. ${ }^{38}$ Chinetti et al found both adipoR1 and adipoR 2 to be present in atherosclerotic human lesions, with a particularly constant amount of adipoR 2 in activated macrophages. ${ }^{39}$ This may explain the detection of the adipoR2 signal in our studies inside macrophage-loaded atherosclerotic plaques, but at the same time this illustrates that the binding of gAd in the outer layer of the atherosclerotic plaques is independent of adipoR2. Like adipoR2, the calreticulin signal was detected in the plaques inside and surrounding the cell nuclei. It is known that calreticulin is a multifunctional protein that acts as a major $\mathrm{Ca}^{2+}$-binding protein in the lumen of the endoplasmic reticulum. It was also found in the nucleus, suggesting that it may have a role in transcription regulation. ${ }^{40}$ Regarding the data by Takemura et $\mathrm{al}^{24}$ showing very detailed interactions between fAd and calreticulin in their in vitro studies, we found that the strong gAd signal, which was observed in our ex vivo experimental model in the surface area of the plaques cannot be generated through a direct interaction with calreticulin, which is detected beneath and not on the surface. Further, the adiponectin binding receptor, T-cadherin, is known to be upregulated in atherosclerotic lesions.$^{38}$ However, because T-cadherin is considered to be a receptor for the medium molecular weight and high molecular weight adiponectin subfractions and not for $\mathrm{gAd}^{23}$ this protein is not likely to be responsible for the intense gAd signal observed at the surface of atherosclerotic plaques.

Thus, the reason why the binding efficiency of gAd to atherosclerotic lesions is much higher compared with fAd-Sfs is not clear at all. We speculate that it could be a matter of molecular size. While the circulating amount of fAd contains subfractions larger than $60 \mathrm{kDa}$ (originating from the trimeric form), the cleaved globular domain has only $17 \mathrm{kDa}$ and can therefore better diffuse through the fenestrated cellular membrane of the injured aortic endothelium. However, this theory remains to be proven in further in vitro studies.

In conclusion, we have outlined distinct differences in the binding efficiency of fAd and gAd to atherosclerotic lesions. We propose that the high binding efficiency of gAd to atherosclerotic plaques represents an inflammation-based, mainly receptor-independent, process of accumulation of gAd in the fibrous cap of atherosclerotic plaques. For that reason, and due to the fact that blood levels of gAd are much lower compared with the levels of fAd-Sfs, ${ }^{16} \mathrm{gAd}$ seems to be an appropriate targeting sequence. By using an optimized "noninflammatory" gAd concentration, gAd could be exploited for the imaging of atherosclerotic plaques and possibly in therapeutic strategies against emerging atherosclerosis, before fatal clinical symptoms like plaque rupture and thrombosis occur.

\section{Acknowledgments}

The authors thank Tatjana Kueznik for directing the confocal laser scanning microscopic imaging, and Heidi Kratzer 
and Dr Andreas Zimmer for their technical support. This work was supported by the Austrian Nano-Initiative, which cofinanced this work as part of the NanoHealth project, in turn financed by the Austrian Research Promotion Agency.

\section{Disclosure}

The authors declare that they have no competing interests or other interests that might be perceived to influence the results and discussion reported in this paper.

\section{References}

1. Kershaw EE, Flier JS. Adipose tissue as an endocrine organ. $J$ Clin Endocrinol Metab. 2004;89(6):2548-2556.

2. Friedman JM. Obesity in the new millennium. Nature. 2000;404(6778): 632-634.

3. Maeda K, Okubo K, Shimomura I, Funahashi T, Matsuzawa Y, Matsubara K. cDNA cloning and expression of a novel adipose specific collagen-like factor, apM1 (AdiPose Most abundant gene transcript 1). Biochem Biophys Res Commun. 1996;221(2):286-289.

4. Scherer PE, Williams S, Fogliano M, Baldini G, Lodish HF. A novel serum protein similar to $\mathrm{C} 1 \mathrm{q}$, produced exclusively in adipocytes. J Biol Chem. 1995;270(45):26746-26749.

5. Chandran M, Phillips SA, Ciaraldi T, Henry RR. Adiponectin: more than just another fat cell hormone? Diabetes Care. 2003;26(8):2442-2450.

6. Arita Y, Kihara S, Ouchi N, et al. Paradoxical decrease of an adiposespecific protein, adiponectin, in obesity. Biochem Biophys Res Commun. 1999;257(1):79-83.

7. Mangge H, Almer G, Haj-Yahya S, et al. Nuchal thickness of subcutaneous adipose tissue is tightly associated with an increased LMW/total adiponectin ratio in obese juveniles. Atherosclerosis. 2009; 203(1):277-283.

8. Mangge H, Almer G, Haj-Yahya S, et al. Preatherosclerosis and adiponectin subfractions in obese adolescents. Obesity (Silver Spring). 2008;16(12):2578-2584.

9. Bobbert T, Rochlitz H, Wegewitz U, et al. Changes of adiponectin oligomer composition by moderate weight reduction. Diabetes. 2005; 54(9):2712-2719.

10. Yamauchi T, Kamon J, Waki H, et al. Globular adiponectin protected $\mathrm{ob} / \mathrm{ob}$ mice from diabetes and ApoE-deficient mice from atherosclerosis. J Biol Chem. 2003;278(4):2461-2468.

11. Li CJ, Sun HW, Zhu FL, et al. Local adiponectin treatment reduces atherosclerotic plaque size in rabbits. J Endocrinol. 2007;193(1): $137-145$.

12. Ouchi N, Ohishi M, Kihara S, et al. Association of hypoadiponectinemia with impaired vasoreactivity. Hypertension. 2003;42(3):231-234.

13. Shimabukuro M, Higa N, Asahi T, et al. Hypoadiponectinemia is closely linked to endothelial dysfunction in man. J Clin Endocrinol Metab. 2003;88(7):3236-3240.

14. Hitsumoto T, Takahashi M, Iizuka T, Shirai K. Relationship between metabolic syndrome and early stage coronary atherosclerosis. J Atheroscler Thromb. 2007;14(6):294-302.

15. Waki H, Yamauchi T, Kamon J, et al. Generation of globular fragment of adiponectin by leukocyte elastase secreted by monocytic cell line THP-1. Endocrinology. 2005;146(2):790-796.

16. Fruebis J, Tsao TS, Javorschi S, et al. Proteolytic cleavage product of 30-kDa adipocyte complement-related protein increases fatty acid oxidation in muscle and causes weight loss in mice. Proc Natl Acad Sci U S A. 2001;98(4):2005-2010.

17. Bruce CR, Mertz VA, Heigenhauser GJ, Dyck DJ. The stimulatory effect of globular adiponectin on insulin-stimulated glucose uptake and fatty acid oxidation is impaired in skeletal muscle from obese subjects. Diabetes. 2005;54(11):3154-3160.
18. Palanivel R, Fang X, Park M, et al. Globular and full-length forms of adiponectin mediate specific changes in glucose and fatty acid uptake and metabolism in cardiomyocytes. Cardiovasc Res. 2007;75(1): $148-157$.

19. Sun Y, Xun K, Wang C, et al. Adiponectin, an unlocking adipocytokine. Cardiovasc Ther. 2009;27(1):59-75.

20. Sun Y, Chen X. Effect of adiponectin on apoptosis: proapoptosis or antiapoptosis? Biofactors. 2010;36(3):179-186.

21. Araki S, Dobashi K, Kubo K, Asayama K, Shirahata A. High molecular weight, rather than total, adiponectin levels better reflect metabolic abnormalities associated with childhood obesity. J Clin Endocrinol Metab. 2006;91(12):5113-5116.

22. Yamauchi T, Kamon J, Ito Y, et al. Cloning of adiponectin receptors that mediate antidiabetic metabolic effects. Nature. 2003;423(6941): 762-769.

23. Hug C, Wang J, Ahmad NS, Bogan JS, Tsao TS, Lodish HF. T-cadherin is a receptor for hexameric and high-molecular-weight forms of Acrp30/adiponectin. Proc Natl Acad Sci U S A. 2004;101(28): 10308-10313.

24. Takemura Y, Ouchi N, Shibata R, et al. Adiponectin modulates inflammatory reactions via calreticulin receptor-dependent clearance of early apoptotic bodies. J Clin Invest. 2007;117(2): 375-386.

25. Okamoto Y, Arita Y, Nishida M, et al. An adipocyte-derived plasma protein, adiponectin, adheres to injured vascular walls. Horm Metab Res. 2000;32(2):47-50.

26. Ouchi N, Kihara S, Arita Y, et al. Adipocyte-derived plasma protein, adiponectin, suppresses lipid accumulation and class A scavenger receptor expression in human monocyte-derived macrophages. Circulation. 2001;103(8):1057-1063.

27. Rohde E, Bartmann C, Schallmoser K, et al. Immune cells mimic the morphology of endothelial progenitor colonies in vitro. Stem Cells. 2007;25(7):1746-1752.

28. Reinisch A, Hofmann NA, Obenauf AC, et al. Humanized large-scale expanded endothelial colony-forming cells function in vitro and in vivo. Blood. 2009;113(26):6716-6725.

29. Rohde E, Malischnik C, Thaler D, et al. Blood monocytes mimic endothelial progenitor cells. Stem Cells. 2006;24(2):357-367.

30. Libby P, Nahrendorf M, Weissleder R. Molecular imaging of atherosclerosis: a progress report. Tex Heart Inst J. 2010;37(3): 324-327.

31. Kamio N, Akifusa S, Yamaguchi N, Nonaka K, Yamashita Y. Antiinflammatory activity of a globular adiponectin function on RAW 264 cells stimulated by lipopolysaccharide from Aggregatibacter actinomycetemcomitans. FEMS Immunol Med Microbiol. 2009;56(3): 241-247.

32. Zhang P, Wang Y, Fan Y, Tang Z, Wang N. Overexpression of adiponectin receptors potentiates the antiinflammatory action of subeffective dose of globular adiponectin in vascular endothelial cells. Arterioscler Thromb Vasc Biol. 2009;29(1):67-74.

33. Hattori Y, Hattori S, Kasai K. Globular adiponectin activates nuclear factor-kappaB in vascular endothelial cells, which in turn induces expression of proinflammatory and adhesion molecule genes. Diabetes Care. 2006;29(1):139-141.

34. Tsatsanis C, Zacharioudaki V, Androulidaki A, et al. Adiponectin induces TNF-alpha and IL-6 in macrophages and promotes tolerance to itself and other pro-inflammatory stimuli. Biochem Biophys Res Commun. 2005;335(4):1254-63.

35. Shapiro L, Scherer PE. The crystal structure of a complement-1q family protein suggests an evolutionary link to tumor necrosis factor. Curr Biol. 1998;8(6):335-338.

36. Tomizawa A, Hattori Y, Kasai K, Nakano Y. Adiponectin induces NF-kappaB activation that leads to suppression of cytokine-induced NF-kappaB activation in vascular endothelial cells: globular adiponectin vs high molecular weight adiponectin. Diab Vasc Dis Res. 2008;5(2): 123-127. 
37. Park PH, Huang H, McMullen MR, Mandal P, Sun L, Nagy LE. Suppression of lipopolysaccharide-stimulated tumor necrosis factoralpha production by adiponectin is mediated by transcriptional and post-transcriptional mechanisms. J Biol Chem. 2008;283(40): 26850-26858.

38. Takeuchi T, Adachi Y, Ohtsuki Y, Furihata M. Adiponectin receptors, with special focus on the role of the third receptor, T-cadherin, in vascular disease. Med Mol Morphol. 2007;40(3):115-120.
39. Chinetti G, Zawadski C, Fruchart JC, Staels B. Expression of adiponectin receptors in human macrophages and regulation by agonists of the nuclear receptors PPARalpha, PPARgamma, and LXR. Biochem Biophys Res Commun. 2004;314(1):151-158.

40. Michalak M, Corbett EF, Mesaeli N, Nakamura K, Opas M. Calreticulin: one protein, one gene, many functions. Biochem J. 1999; 344( Pt 2):281-292.

\section{Publish your work in this journal}

Biologics: Targets \& Therapy is an international, peer-reviewed journal focusing on the patho-physiological rationale for and clinical application of Biologic agents in the management of autoimmune diseases, cancers or other pathologies where a molecular target can be identified This journal is indexed on PubMed Central, CAS, EMBase, Scopus

\section{Dovepress}

and the Elsevier Bibliographic databases. The manuscript management system is completely online and includes a very quick and fair peerreview system, which is all easy to use. Visit http://www.dovepress. com/testimonials.php to read real quotes from published authors. 